\title{
ATPase inhibitory factor 1 inhibition improves the antitumor of YC-1 against hepatocellular carcinoma
}

\author{
XUEMEI DING, JIAN KONG, WENLEI XU, SHUYING DONG, YINGRUI DU, \\ CHANGYU YAO, JUN GAO, SHAN KE, SHAOHONG WANG and WENBING SUN
}

\begin{abstract}
Department of Hepatobiliary Surgery, Beijing Chao-yang Hospital, Capital Medical University, Beijing 100043, P.R. China
\end{abstract}
Received January 14, 2018; Accepted July 27, 2018

DOI: $10.3892 / \mathrm{ol} .2018 .9266$

\begin{abstract}
YC-1 is a synthetic compound, which serves as a hypoxia-inducible factor 1- $\alpha$ inhibitor or sensitizer to enhance the effect of chemotherapy. Previous studies have revealed the anti-cancer effects of YC-1 in various types of cancer, including hepatocellular carcinoma (HCC). ATPase inhibitory factor 1 (IF1) is upregulated in a number of human carcinomas and regulates mitochondrial bioenergetics and structure. However, whether IF1 is involved in the antitumor effects of YC-1 against HCC remains unclear. The present study examined the function of IF1 in HCC and its potential role in YC-1 effects within HCC cells. MTT, colony formation and Transwell assays revealed that IF1 overexpression promoted proliferation, colony formation and invasion of HCC cells, while IF1 downregulation had the opposite effects. Overexpression of IF1 reversed the inhibitory effects of $\mathrm{YC}-1$ on Huh7 cell growth and invasion activities, while downregulation of IF1 increased the sensitivity of HCCLM3 cells to YC-1. YC-1 treatment of HCCLM3 and Huh7 cells reduced the levels of phosphorylated (p-) signal transducer and activator of transcription 3 (STAT3) and IF1, and increased the expression of E-cadherin. IF1 knockdown resulted in decreased p-STAT3 levels and increased E-cadherin expression, while IF1 overexpression increased p-STAT3 levels and reduced the expression of E-cadherin. The present study demonstrated that the inhibition of IF1 improves the antitumor effects of YC-1 in HCC cells. These findings support the clinical strategy of combining YC-1 and an IF1 inhibitor for the treatment of HCC.
\end{abstract}

\section{Introduction}

Hepatocellular carcinoma (HCC) is the fifth most common malignancy and the second leading cause of cancer-related

Correspondence to: Dr Wenbing Sun, Department of Hepatobiliary Surgery, Beijing Chao-yang Hospital, Capital Medical University, 5 Jingyuan Road, Beijing 100043, P.R. China

E-mail: cyhswb@qq.com

Key words: YC-1, adenosine triphosphatase inhibitory factor 1, hepatocellular carcinoma, invasion deaths worldwide (1). Potentially curative treatment strategies such as tumor resection, liver transplantation, or local ablation are only limited to HCC at early stage (2). Patients with advanced stage HCC show poor responses to traditional chemotherapy and sorafenib is the only approved systemic therapy for advanced HCC, however, the response rate is quite low $(3,4)$. Therefore, new therapeutic strategies for HCC treatment are urgently needed.

1-benzyl-3-(5'-hydroxymethyl-2'-furyl) indazole (YC-1) is a synthetic compound that exhibits various potent biological and pathological activities, including antiplatelet activity, soluble guanylyl cyclase activity, suppression of hypoxia-induced factor-1 $\alpha$ (HIF-1 $\alpha)$, and anti-cancer activity (5). A previous report showed that $\mathrm{YC}-1$ exerts antitumor effects in $\mathrm{HCC}$ through suppressing HIF-1 $\alpha$ and inducing $\mathrm{S}$ cell cycle arrest or G0-G1 cell cycle arrest (6,7). YC-1 also enhanced chemosensitivity in HCC via inhibition of signal transducer and activator of transcription (STAT3) activity (8). Our previous study also suggested that YC-1 enhanced the antitumor activity of sorafenib through STAT3 in HCC (9). These studies suggest that YC-1 acts as an HIF-1 $\alpha$ inhibitor or sensitizer to enhance the effect of chemotherapeutics, however, the precise mechanisms underlying the antitumor effects of YC-1 against HCC have not been fully elucidated.

ATPase inhibitory factor 1 (IF1) is encoded by the ATPIF1 gene that is located in chromosomes 1 and 4 of human and mouse genomes, respectively (10). IF1 is implicated in the control of both mitochondrial bioenergetics and structure by regulating the activity and oligomerization of the F1Fo-ATPsynthase (hereinafter referred to as ATP synthase) (11-16). The function of IF1 as an ATP synthase inhibitor is regulated by matrix $\mathrm{pH}$ under conditions of mitochondrial de-energization and by the phosphorylation of S39 under several physiological situations, such as progression through the cell cycle, hypoxia, and rapid changes in metabolic demand (11). A previous study demonstrated that overexpression of HIF-1 $\alpha$ by hypoxia or $\mathrm{CoCl}_{2}$ treatment augmented IF1 protein levels in a rat hepatic epithelial cell line (17). The pathway may be suitable to the mechanism of IF1 in HCC. Some studies showed that IF is upregulated in many human carcinomas and the level of IF1 expression in HCC, gliomas, and gastric cancers correlates with aggressiveness and invasiveness of tumors and poor prognosis in patients $(14,18)$. Whether IF1 is involved in the antitumor effect of YC-1 against HCC remains unclear. 
In the present study, we examined the function of IF1 in HCC cells and the potential role or involvement of IF1 in the antitumor effects of YC-1 in the HCCLM3 and Huh7 HCC cell lines in vitro.

\section{Materials and methods}

Cell lines and cell culture. Human HCC cell line Huh7 was obtained from the American Type Culture Collection (Manassas, VA, USA). The human HCC cell line HCCLM3 was obtained from the China Center for Type Culture Collection and Cell Bank of the Chinese Academy of Sciences (Shanghai, China). Cells were maintained in high-glucose Dulbecco's modified Eagle's medium (DMEM) supplemented with $10 \%$ fetal bovine serum (FBS), $100 \mathrm{U} / \mathrm{ml}$ penicillin and $100 \mu \mathrm{g} / \mathrm{ml}$ streptomycin (Thermo Fisher Scientific, Inc. Waltham, MA, USA) in a humidified atmosphere of $5 \% \mathrm{CO}_{2}$ at $37^{\circ} \mathrm{C}$. Cell lines were immediately expanded and frozen down such that cell lines could be restarted every 3 months from a frozen vial of the same batch of cells. No further authentication was done. All cell lines were routinely tested to rule out mycoplasma contamination. YC-1 was obtained from Sigma-Aldrich (Merck KGaA, Darmstadt, Germany).

Establishment of stable knockdown or overexpression cell lines. Lentiviral vectors encoding the human IF1 gene (NM_016311.4) and shRNA-IF1 (CACCATGAAGAAGAAATCGTT) were constructed by Beijing LiKeli BioTECH Co. Ltd. (Beijing, China) and designated as LV-IF1 and LV-shRNA-IF1, respectively. The empty vectors (LV-Ctrl or LV-shRNA-Ctrl) were used as a negative control. The lentiviral vectors were transfected into HCC cells with a multiplicity of infection of 20 to 30 in the presence of polybrene $(2 \mu \mathrm{g} / \mathrm{ml})$. At $48 \mathrm{~h}$ after transfection, transfected cells were selected for 2 weeks with $2 \mu \mathrm{g} / \mathrm{ml}$ puromycin (Sigma-Aldrich; Merck KGaA). Pooled populations of knockdown cells and overexpression cells, which were obtained 2 weeks after drug selection without subcloning, were used in in vitro experiments.

Proliferation assay. Cell proliferation was analyzed using the 3-(4,5-dimethylthiazol-2-yl)-2,5-diphenyltetrazolium bromide (MTT) assay. Briefly, HCC cells were seeded in 96-well plates at a density of $3 \times 10^{3}$ cells/well and treated with various concentrations of YC-1 for 24,48 , or $72 \mathrm{~h}$. MTT solution was added to each well at a final concentration of $0.5 \mathrm{mg} / \mathrm{ml}$ and cells were incubated for $4 \mathrm{~h}$. Formazan crystals resulting from MTT reduction were then dissolved by the addition of $150 \mu 1$ dimethyl sulfoxide per well. The absorbance was measured at $570 \mathrm{~nm}$ using an automated ELISA plate reader.

Colony formation assay. Six-well dishes were seeded with $1 \times 10^{3}$ cells and cells were cultured for $24 \mathrm{~h}$. The cells were then incubated in the presence of various concentrations of YC-1 for $24 \mathrm{~h}$ in complete medium, washed with media, and allowed to grow in complete medium for 2 weeks. The obtained colonies were washed with PBS, fixed in $4 \%$ paraformaldehyde for 20 min at room temperature, washed with PBS, and then stained with crystal violet. The stained colonies were then counted.

Invasion assay. Cell invasion assays were performed using a modified Boyden chamber (Costar; Corning Inc., Corning, NY, USA) that was precoated with Matrigel. HCCLM3 or Huh7 cells ( $2 \times 10^{4}$ cells per well) and $10 \mu \mathrm{M}$ YC-1 were added into the upper chamber, and $600 \mu \mathrm{l}$ DMEM with $10 \%$ FBS were added into the lower chamber. The chambers were incubated for $24 \mathrm{~h}$. After removing the filter inserts and the cells on the upper side of the filter, the migrated cells on the lower chamber were stained with crystal violet for $20 \mathrm{~min}$, washed with PBS, and photographed under an inverted fluorescence microscope (Olympus IX51) equipped with an Olympus Qcolor 3 digital camera (both Olympus Corp., Tokyo, Japan). Migration was assessed by counting the number of stained cells from 5 random fields at magnification, $\mathrm{x} 10$.

Western blot analysis. Equivalent amounts of whole cell extracts were subjected to SDS-PAGE and transferred to nitrocellulose membranes. The membranes were blocked with $5 \%$ non-fat milk for $2 \mathrm{~h}$ and then incubated with primary antibodies overnight at $4{ }^{\circ} \mathrm{C}$, followed by incubation with the appropriate HRP-conjugated secondary antibody for $1.5 \mathrm{~h}$ at room temperature. Immunoreactivity was detected with SuperSignal West Pico substrate (Thermo Fisher Scientific, Inc.) according to the manufacturer's instructions. Primary antibodies anti-IF1, anti-E-cadherin, anti-phosphorylated (p-) STAT3, and anti- $\beta$-actin were obtained from CST (Danvers, MA, USA). Horseradish peroxidase (HRP)-labeled anti-mouse and anti-rabbit secondary antibodies were from Santa Cruz Biotechnology, Inc. (Dallas, TX, USA). All other antibodies were purchased from Abcam (Cambridge, MA, USA).

Reverse transcription-quantitative polymerase chain reaction $(R T-q P C R)$. Total mRNA was extracted using the TRIzol reagent (Invitrogen; Thermo Fisher Scientific, Inc.) and RT was performed using an RT-PCR kit (TransGen Biotech Co., Ltd., Beijing, China). qPCR experiments were conducted on an DNA Engine Opticon System (MJ Research Inc.; Bio-Rad Laboratories, Inc.) using SYBR-Green PCR Master Mix kit in triplicate specific primers. The sequences of primers to determine the expression of the target gene were listed as follows: IF1 forward, 5'-GGGCCTTCGGAAAGAGAG-3' and reverse, 5'-TTCAAAGCTGCCAGTTGTTC-3'; and glyceraldehydes 3-phosphate dehydrogenase (GAPDH) forward, 5'-CGG AGTCAACGGATTTGGTCGTAT-3' and reverse, 5'-AGCCTT CTCCATGGTGGTGAAGAC-3'. The PCR thermocycling conditions consisted of $5 \mathrm{~min}$ at $95^{\circ} \mathrm{C}$ followed by 40 cycles of denaturation for $30 \mathrm{sec}$ at $95^{\circ} \mathrm{C}$, annealing for $30 \mathrm{sec}$ at $56^{\circ} \mathrm{C}$ and a primer extension for $30 \mathrm{sec}$ at $72^{\circ} \mathrm{C}$. Relative gene expression levels were quantified using the $2^{-\Delta \Delta C q}$ method (19).

Statistical analysis. All values are expressed as the mean \pm standard error of the mean. The data were analyzed using Student's t-test or the analysis of variance test with Tukey's post hoc test. $\mathrm{P}<0.05$ was considered to indicate a statistically significant difference. GraphPad Prism 5 (GraphPad Software Inc., San Diego, CA, USA) was used for these analyses.

\section{Results}

IF1 regulates the proliferation and invasion of HCC cells. To investigate the function of IF1 in HCC, we first examined the expression of IF1 in HCCLM3 and Huh7 cell lines. Western 
A

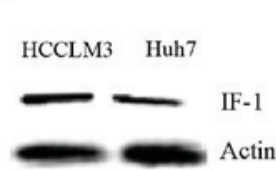

B

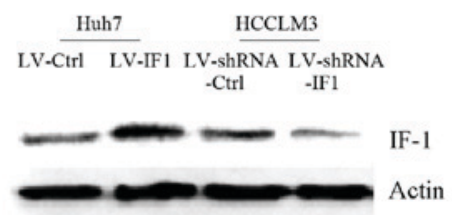

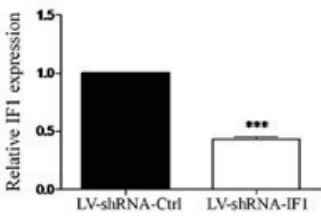

D

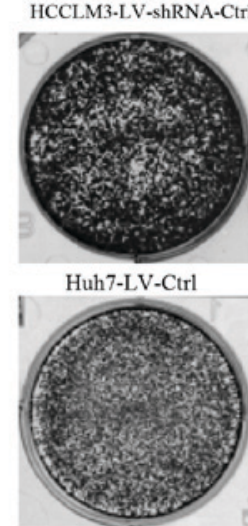

HCCLM3-LV-shRNA-IF 1

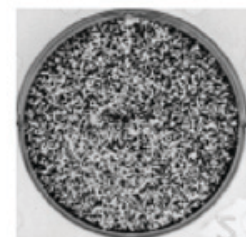

Huh7-LV-IF1

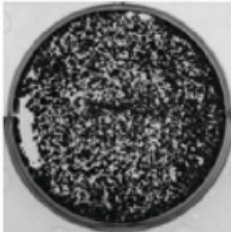

C

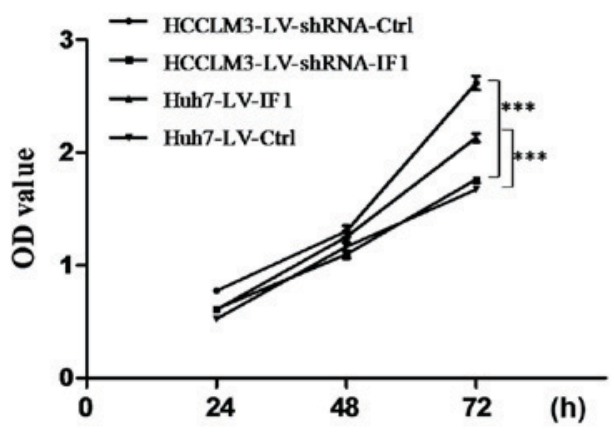

E HCCLM3-LV-shRNA-Ctrl
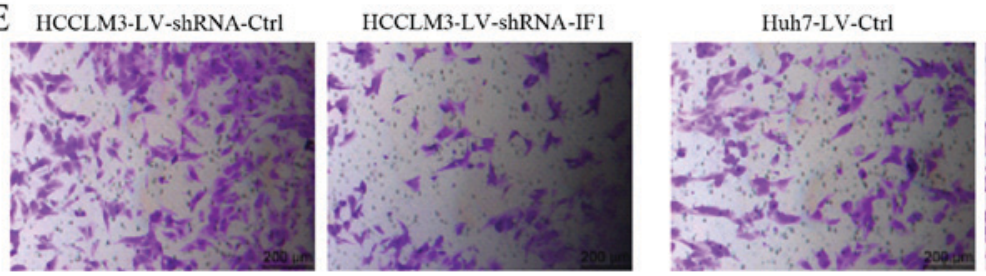

Huh7-LV-IF 1

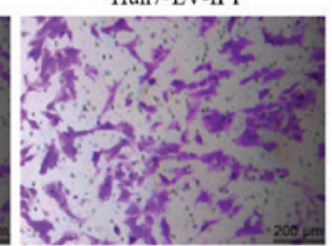

Figure 1. IF1 regulates proliferation and invasion of HCC cells. (A) Western blot analysis of IF1 in Huh7 and HCCLM3 cells. Actin was used as loading control. (B) Lentiviral overexpression of IF1 in Huh7 cells (Huh7-LV-IF1 or Huh7-LV-Ctrl) or IF1 shRNA in HCCLM3 cells (HCCLM3-shRNA-IF1 or HCCLM3-shRNA-Ctrl). Western blot analysis and RT-qPCR were performed for the indicated proteins and genes. ${ }^{* * *}$ P<0.001 vs. LV-shRNA-Ctrl. (C) MTT assays, (D) colony formation assays and (E) invasion assays of HCC cells with IF1 overexpression or IF1 shRNA knockdown compared with the appropriate controls (scale bars, $200 \mu \mathrm{m}$ ). ${ }^{* * *} \mathrm{P}<0.001$, as indicated. IF1, inhibitory factor 1; HCC, hepatocellular carcinoma; shRNA, short hairpin RNA; Ctrl, control; OD, optical density; MTT, 3-(4,5-dimethylthiazol-2-yl)-2,5-diphenyltetrazolium bromide.

blot analysis revealed that the expression of IF1 was higher in HCCLM3 cells compared with Huh7 cells (Fig. 1A). To examine the effects of altered IF1 expression, we transfected a lentiviral expression vector that expresses IF1 in Huh7 cells (Huh7-LV-IF1) or shRNA for IF1 knockdown in HCCLM3 cells (HCCLM3-shRNA-IF1) and generated stable cell lines, together with the appropriate controls (Huh7-LV-Ctrl and HCCLM3-shRNA-Ctrl) and RT-qPCR was used to confirm the results (Fig. 1B).

We next performed a series of assays to clarify the function of IF1 in HCC cells. We found that knockdown of IF1 could significantly inhibit the proliferation, colony formation and invasion activities of HCCLM3 cells compared with controls (Fig. 1C-E). In contrast, overexpression of IF1 in Huh7 cells resulted in increased proliferation, colony formation and invasion (Fig. 1C-E). Together these results indicated a role for IF1 in regulating proliferation and invasion of HCC cells.

Overexpression of IF1 reduced the inhibitory effects of $Y C-1$ in Huh7 cells. We next examined whether IF1 could impact the effects of YC-1 on HCC cells using Huh7-LV-IF1 and Huh7-LV-Ctrl cells. Consistent with previous studies on YC-1 anti-cancer effects, we found that YC-1 treatment reduced the proliferation, colony formation and invasion activities of Huh7 cells (Fig. 2). However, Huh7 cells with overexpression of IF1 showed reduced sensitivity to the negative effects of YC-1 on proliferation and attenuated the YC-1-induced inhibition of invasion (Fig. 2).

Knockdown of IF1 elevated the sensitivity of HCCLM3 cells to $Y C-1$. We next evaluated the effect of IF1 knockdown on the sensitivity of HCC cells to YC-1. We found that HCCLM3 cells with knockdown of IF1 showed elevated sensitivity to the inhibitory effects of YC-1 on cell proliferation, colony formation and invasion activities compared with cells transfected with the shRNA control (Fig. 3).

STAT3 activation is involved in the effect of IF 1 on YC-1 treatment in HCC cells. To investigate the underlying mechanism of the role of IF1 in the effect of YC-1 on HCC cells, we examined a panel of several molecular factors by western blot analysis. YC-1 treatment in HCCLM3 and Huh7 cells decreased the expression of p-STAT3 and IF1 and increased the expression of E-cadherin (Fig. 4). IF1 knockdown significantly reduced the levels of p-STAT3 and increased the expression of E-cadherin, while IF1 overexpression increased the expression of p-STAT3 and decreased the expression of E-cadherin (Fig. 4).

\section{Discussion}

In the present study, we provided evidence that IF1 plays an important role in the antitumor effects of YC-1 in HCC. Our 
A

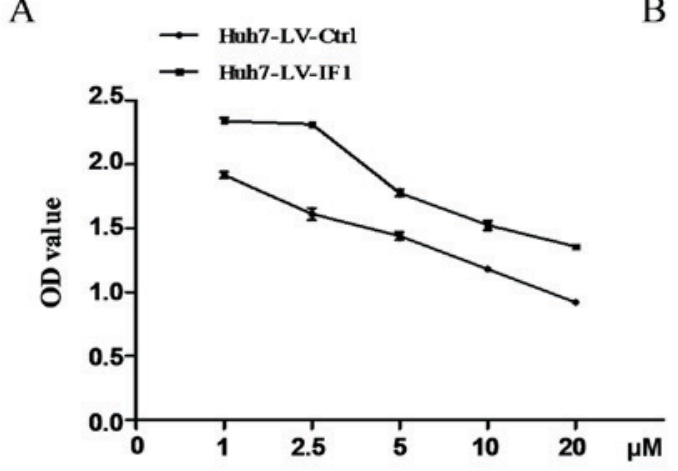

$\mathrm{C}$

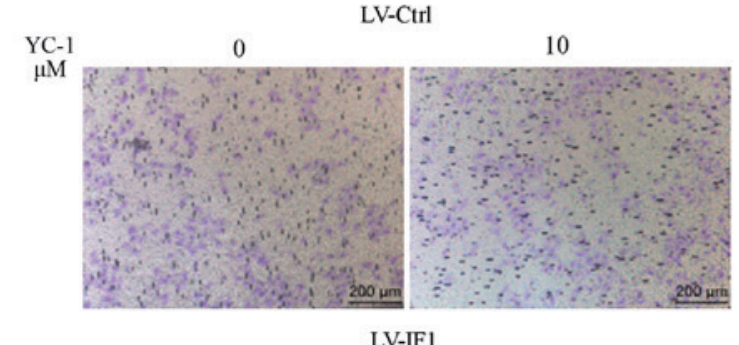

0

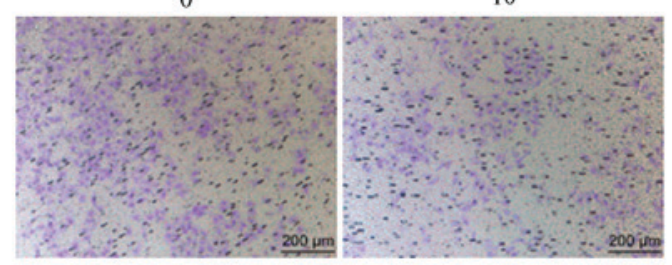

B LV-Cur
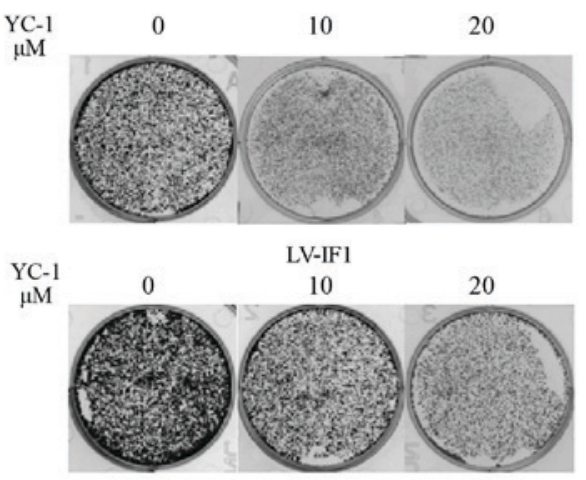

Figure 2. IF1 overexpression reverses the inhibitory effects of YC-1 in Huh7 cells. (A) MTT assays of Huh7 cells with IF1 overexpression or controls treated with various concentrations of YC-1 for $24 \mathrm{~h}$. (B) Colony formation assays in Huh7 cells with IF1 overexpression or controls cultured with 10 or $20 \mu \mathrm{M}$ YC-1 for 14 days. (C) Invasion assays of Huh7 cells with IF1 overexpression or controls in the presence of $10 \mu \mathrm{M}$ YC-1 for $24 \mathrm{~h}$ (scale bars, $200 \mu \mathrm{m}$ ). IF1, inhibitory factor 1; Ctrl, control; OD, optical density; YC-1, 1-benzyl-3-(5'-hydroxymethyl-2'-furyl) indazole.

results demonstrated that IF1 was involved in the proliferation, colony formation and invasion activities of $\mathrm{HCC}$ cells. Overexpression of IF1 reversed the inhibitory effects of YC-1 in Huh7 cells, and knockdown of IF1 elevated the sensitivity of HCCLM3 cells to YC-1. STAT3 signaling pathways may also be involved in the process. Our results suggest that inhibition of IF1 could improve the antitumor effects of YC-1 against HCC.

IF1 is an inhibitor of the mitochondrial $\mathrm{H}(+)$-ATP synthase, and several studies have suggested that IF1 is involved in tumor progression. IF1 was shown to be an independent prognostic factor in non-small cell lung cancer (13). In the liver, IF1 could downregulate oxidative phosphorylation and induce a tumor-promoting metabolic state (20). Another study showed that IF1 was a prognostic marker in gastric cancer and that IF1 contributes to proliferation and invasion of human gastric cancer cells (14). Silencing of IF1 in bladder cancer inhibited cell growth via cell cycle arrest (15). A study in HCC showed that a reciprocal activation between $\mathrm{IF} 1$ and $\mathrm{NF}-\kappa \mathrm{B}$ drives angiogenesis and metastasis (16). Analysis of IF1 expression in tumors in breast and colon cancer patient cohorts revealed its relevance as a predictive marker for clinical outcome (21). Another report showed that upregulation of IF1 in human tumors mediated the metabolic shift of cancer cells to a Warburg phenotype (22). Our results also showed that knockdown of IF1 inhibited the proliferation, colony formation and invasion activities of HCC cells, while IF1 overexpression had the opposite effect.
YC-1 functions to inhibit HIF-1 $\alpha$ and suppress tumor progression (23), and the direct inhibiting growth of tumor is limit. Indeed, resistance to YC-1 necessitates the use of increased therapeutic doses and can result in increased adverse side effects. A previous study on HCC cells showed that $\mathrm{YC}-1$ exhibited an antiproliferative effect and arrests the cell cycle in $\mathrm{G0}-\mathrm{G} 1$, however, the $\mathrm{IC}_{50}$ value was about $46 \mu \mathrm{M}$ (24). Notably, our previous study demonstrated that YC-1 enhanced the antitumor activity of sorafenib through inhibition of STAT3 in HCC (9). Another report showed that YC-1 could potentiate the apoptotic effect of licochalcone A on human epithelial ovarian carcinoma cells via activation of death receptor and mitochondrial pathways (25). These studies suggest YC-1 could be also used as a sensitizer to enhance the effect of chemotherapeutics. Our current results demonstrated that increased IF1 expression reduced the inhibitory effects of YC-1 and knockdown of IF1 enhanced the growth and invasion suppressive effects of YC-1 in HCC cells. These findings indicate that IF1 may be involved in the effects of YC-1 on HCC cells and suggest that a combination of silencing IF1 and YC-1 may be a useful strategy for HCC treatment.

STAT3 is a central mediator of cancer metastasis and a target of anticancer drugs for blocking tumor metastasis. STAT3 is activated by its binding to various ligands, such as interleukin-6 (IL-6), interferon, and IL-10 (26-29). Numerous 

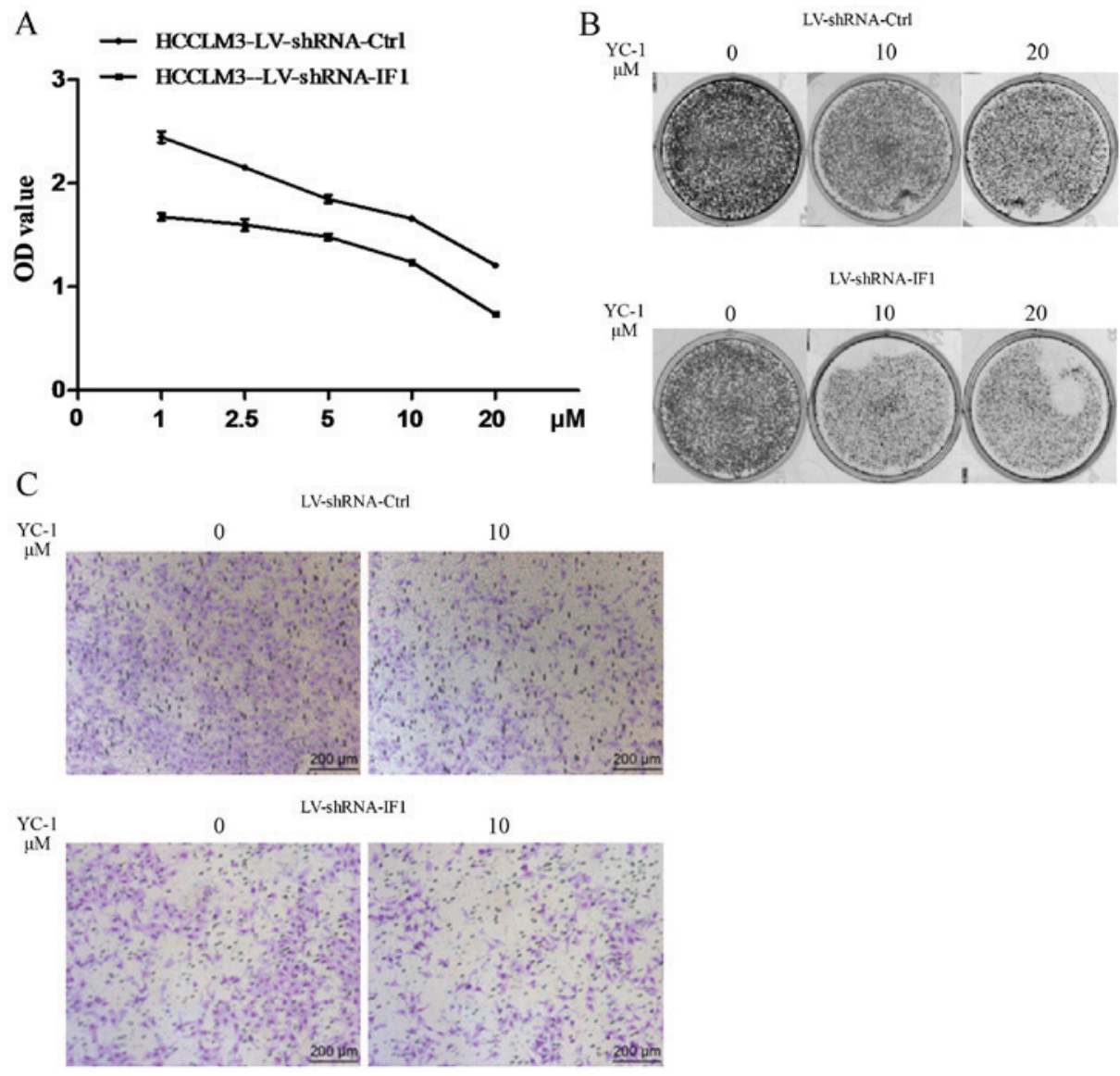

Figure 3. Knockdown of IF1 increases the sensitivity of HCCLM3 cells to YC-1. (A) MTT assays of HCCLM3 cells with IF1 knockdown or control shRNA in the presence of various concentrations of YC-1 for $24 \mathrm{~h}$. (B) Colony formation assays in HCCLM3 cells with IF1 knockdown or control shRNA in the presence of 10 or $20 \mu \mathrm{M}$ YC-1 for 14 days. (C) Invasion assays of HCCLM3 cells with IF1 knockdown or control shRNA in the presence of $10 \mu \mathrm{M}$ YC-1 for $24 \mathrm{~h}$ (scale bars, $200 \mu \mathrm{m}$ ). IF1, inhibitory factor 1; shRNA, short hairpin RNA; Ctrl, control; OD, optical density; YC-1, 1-benzyl-3-(5'-hydroxymethyl-2'-furyl) indazole; MTT, 3-(4,5-dimethylthiazol-2-yl)-2,5-diphenyltetrazolium bromide.

A

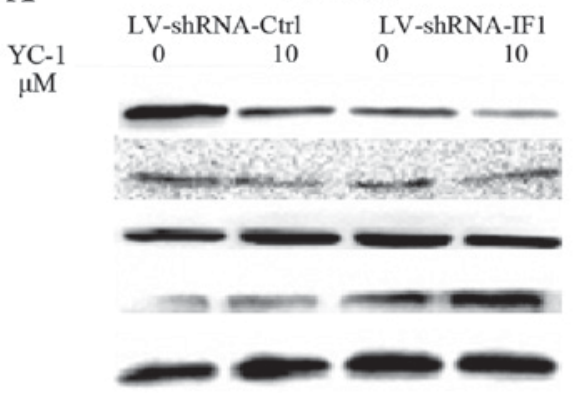

HCCLM3

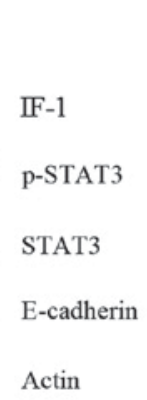

B

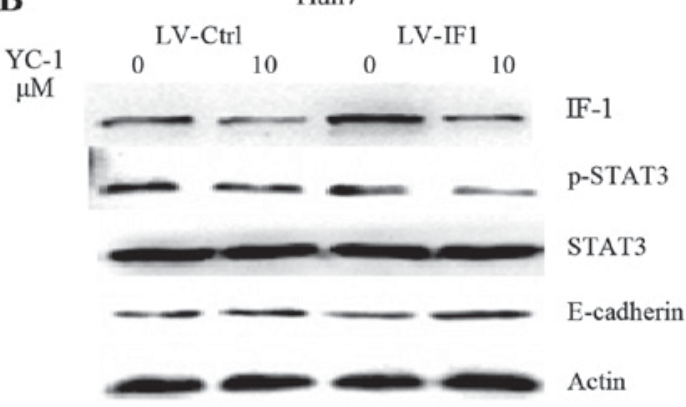

Figure 4. STAT3 is involved in the effect of IF1 in YC-1-induced inhibitory activities in HCC cells. Western blot analysis of the indicated proteins in (A) HCCLM3 cell lines with IF knockdown or control shRNA and (B) Huh7 cell lines with IF1 overexpression or controls treated with $10 \mu \mathrm{M}$ YC-1 for $24 \mathrm{~h}$. Actin was used as a normalization control. IF1, inhibitory factor 1; shRNA, short hairpin RNA; Ctrl, control; STAT3, signal transducer and activator of transcription 3; p-, phosphorylated; YC-1, 1-benzyl-3-(5'-hydroxymethyl-2'-furyl) indazole.

studies have revealed that the JAK/STAT signaling pathway is involved in several aspects of tumorigenesis, including proliferation, apoptosis, angiogenesis, and metastasis $(30,31)$. Activated STAT3 has been reported in various human cancers, and elevated level of activated STAT3 is associated with poor prognosis. Our results showed that overexpression of IF1 could induce the levels of p-STAT3, while IF1 knockdown reduced p-STAT3 levels. Another study showed that YC-1 could inhibit STAT3 activity by enhancing the polyubiquitination of p-STAT3 (705) and overexpression of STAT3 reversed YC-1-induced cell death. YC-1 may also suppress the expression of p-STAT3 through inhibiting SHP-1 activity. Together this suggests that STAT3 may function as a bridge in the interaction between IF1 and YC-1.

In conclusion, our results demonstrate that inhibition of IF1 improves the antitumor effects of YC-1 in HCC cells. This finding supports the clinical development of combining YC-1 and an IF1 inhibitor for the treatment of HCC. 


\section{Acknowledgements}

Not applicable.

\section{Funding}

The present study was supported by the National Natural Science Foundation of China (grant nos. 81572957 and 81502650).

\section{Availability of data and materials}

The datasets used and/or analyzed during the current study are available from the corresponding author on reasonable request.

\section{Authors' contributions}

WS conceived and supervised the study, and helped to draft the manuscript. JG, SW and SK participated in the design of the study. XD and JK performed the experiments and drafted the manuscript. SD, WX, YD and CY analyzed the data. All authors read and approved the final manuscript.

\section{Ethics approval and consent to participate}

Not applicable.

\section{Patient consent for publication}

Not applicable.

\section{Competing interests}

The authors declare that there are no competing interests.

\section{References}

1. Forner A, Llovet JM and Bruix J: Hepatocellular carcinoma. Lancet 379: 1245-1255, 2012.

2. Xu XL, Liu XD, Liang M and Luo BM: Radiofrequency ablation versus hepatic resection for small hepatocellular carcinoma: Systematic review of randomized controlled trials with meta-analysis and trial sequential analysis. Radiology 287 : 461-472, 2018

3. Niu L, Liu L, Yang S, Ren J, Lai PBS and Chen GG: New insights into sorafenib resistance in hepatocellular carcinoma: Responsible mechanisms and promising strategies. Biochim Biophys Acta 1868: 564-570, 2017.

4. Bruix J, Cheng AL, Meinhardt G, Nakajima K, De Sanctis Y and Llovet J: Prognostic factors and predictors of sorafenib benefit in patients with hepatocellular carcinoma: Analysis of two phase III studies. J Hepatol 67: 999-1008, 2017.

5. Chun YS, Yeo EJ and Park JW: Versatile pharmacological actions of YC-1: Anti-platelet to anticancer. Cancer Lett 207: 1-7, 2004.

6. Yeo EJ, Ryu JH, Chun YS, Cho YS, Jang IJ, Cho H, Kim J, Kim MS and Park JW: YC-1 induces $\mathrm{S}$ cell cycle arrest and apoptosis by activating checkpoint kinases. Cancer Res 66: 6345-6352, 2006.

7. Lau CK, Yang ZF, Lam CT, Tam KH, Poon RT and Fan ST: Suppression of hypoxia inducible factor-1alpha (HIF-1alpha) by YC-1 is dependent on murine double minute 2 (Mdm2). Biochem Biophys Res Commun 348: 1443-1448, 2006.

8. Lau CK, Yang ZF, Lam SP, Lam CT, Ngai P, Tam KH, Poon RT and Fan ST: Inhibition of Stat3 activity by YC-1 enhances chemo-sensitivity in hepatocellular carcinoma. Cancer Biol Ther 6: 1900-1907, 2007.
9. Kong J, Kong F, Gao J, Zhang Q, Dong S, Gu F, Ke S, Pan B, Shen Q, Sun H, et al: YC-1 enhances the anti-tumor activity of sorafenib through inhibition of signal transducer and activator of transcription 3 (STAT3) in hepatocellular carcinoma. Mol Cancer 13: 7, 2014

10. García-Bermúdez J and Cuezva JM: The ATPase inhibitory factor 1 (IF1): A master regulator of energy metabolism and of cell survival. Biochim Biophys Acta 1857: 1167-1182, 2016.

11. Garcia-Ledo L, Nuevo-Tapioles C, Cuevas-Martin C, Martínez-Reyes I, Soldevilla B, González-Llorente L and Cuezva JM: Overexpression of the ATPase inhibitory factor 1 favors a non-metastatic phenotype in breast cancer. Front Oncol 7: 69, 2017.

12. Faccenda D, Nakamura J, Gorini G, Dhoot GK, Piacentini M, Yoshida $\mathrm{M}$ and Campanella $\mathrm{M}$ : Control of mitochondrial remodeling by the ATPase inhibitory factor 1 unveils a pro-survival relay via OPA1. Cell Rep 18: 1869-1883, 2017.

13. Gao YX, Chen L, Hu XG, Wu HB, Cui YH, Zhang X, Wang Y, Liu XD and Bian XW: ATPase inhibitory factor 1 expression is an independent prognostic factor in non-small cell lung cancer. Am J Cancer Res 6: 1141-1148, 2016.

14. Yin T, Lu L, Xiong Z, Wei S and Cui D: ATPase inhibitory factor 1 is a prognostic marker and contributes to proliferation and invasion of human gastric cancer cells. Biomed Pharmacother 70: 90-96, 2015.

15. Wei S, Fukuhara H, Kawada C, Kurabayashi A, Furihata M, Ogura S, Inoue K and Shuin T: Silencing of ATPase inhibitory factor 1 inhibits cell growth via cell cycle arrest in bladder cancer. Pathobiology 82: 224-232, 2015.

16. Song R, Song H, Liang Y, Yin D, Zhang H, Zheng T, Wang J, Lu Z, Song X, Pei T, et al: Reciprocal activation between ATPase inhibitory factor 1 and $N F-\kappa B$ drives hepatocellular carcinoma angiogenesis and metastasis. Hepatology 60: 1659-1673, 2014.

17. Huang LJ, Chuang IC, Dong HP and Yang RC: Hypoxia-inducible factor $1 \alpha$ regulates the expression of the mitochondrial ATPase inhibitor protein (IF1) in rat liver. Shock 36: 90-96, 2011.

18. Wu J, Shan Q, Li P, Wu Y, Xie J and Wang X: ATPase inhibitory factor 1 is a potential prognostic marker for the migration and invasion of glioma. Oncol Lett 10: 2075-2080, 2015.

19. Livak KJ and Schmittgen TD: Analysis of relative gene expression data using real-time quantitative PCR and the 2(-Delta Delta C(T)) method. Methods 25: 402-408, 2001.

20. Santacatterina F, Sánchez-Cenizo L, Formentini L, Mobasher MA, Casas E, Rueda CB, Martínez-Reyes I, Núñez de Arenas C, García-Bermúdez J, Zapata JM, et al: Down-regulation of oxidative phosphorylation in the liver by expression of the ATPase inhibitory factor 1 induces a tumor-promoter metabolic state. Oncotarget 7: 490-508, 2016.

21. Sánchez-Aragó M, Formentini L, Martinez-Reyes I, García-Bermudez J, Santacatterina F, Sánchez-Cenizo L, Willers IM, Aldea M, Nájera L, Juarránz A, et al: Expression, regulation and clinical relevance of the ATPase inhibitory factor 1 in human cancers. Oncogenesis 2: e46, 2013.

22. Sánchez-Cenizo L, Formentini L, Aldea M, Ortega AD, García-Huerta $P$, Sánchez-Aragó $M$ and Cuezva JM: Up-regulation of the ATPase inhibitory factor 1 (IF1) of the mitochondrial H+-ATP synthase in human tumors mediates the metabolic shift of cancer cells to a Warburg phenotype. J Biol Chem 285: 25308-25313, 2010.

23. Kong J, Kong J, Pan B, Ke S, Dong S, Li X, Zhou A, Zheng L and Sun WB: Insufficient radiofrequency ablation promotes angiogenesis of residual hepatocellular carcinoma via HIF-1 $\alpha /$ VEGFA. PLoS One 7: e37266, 2012.

24. Wang SW, Pan SL, Guh JH, Chen HL, Huang DM, Chang YL, Kuo SC, Lee FY and Teng CM: YC-1 [3-(5'-Hydroxymethyl-2'-furyl)-1-benzyl Indazole] exhibits a novel antiproliferative effect and arrests the cell cycle in G0-G1 in human hepatocellular carcinoma cells. J Pharmacol Exp Ther 312: 917-925, 2005.

25. Lee CS, Kwak SW, Kim YJ, Lee SA, Park ES, Myung SC, Kim W, Lee MS and Lee JJ: Guanylate cyclase activator YC-1 potentiates apoptotic effect of licochalcone A on human epithelial ovarian carcinoma cells via activation of death receptor and mitochondrial pathways. Eur J Pharmacol 683: 54-62, 2012.

26. Huynh J, Etemadi N, Hollande F, Ernst M and Buchert M: The JAK/STAT3 axis: A comprehensive drug target for solid malignancies. Semin Cancer Biol 45: 13-22, 2017. 
27. Bixel K, Saini U, Kumar Bid H, Fowler J, Riley M, Wanner R, Deepa Priya Dorayappan K, Rajendran S, Konishi I, Matsumura N, et al: Targeting STAT3 by HO3867 induces apoptosis in ovarian clear cell carcinoma. Int J Cancer 141: 1856-1866, 2017.

28. Khan MW, Saadalla A, Ewida AH, Al-Katranji K, Al-Saoudi G, Giaccone ZT, Gounari F, Zhang M, Frank DA and Khazaie K: The STAT3 inhibitor pyrimethamine displays anti-cancer and immune stimulatory effects in murine models of breast cancer. Cancer Immunol Immunother 67: 13-23, 2018.

29. Zeng R, Tang Y, Zhou H, Liu Y, Huang J, Li L, Liu W, Feng Y, Zhou Y, Chen T, et al: STAT3 mediates multidrug resistance of Burkitt lymphoma cells by promoting antioxidant feedback. Biochem Biophys Res Commun 488: 182-188, 2017.
30. Gao P, Niu N, Wei T, Tozawa H, Chen X, Zhang C, Zhang J, Wada Y, Kapron CM and Liu J: The roles of signal transducer and activator of transcription factor 3 in tumor angiogenesis. Oncotarget 8: 69139-69161, 2017.

31. Kim BH, Yi EH and Ye SK: Signal transducer and activator of transcription 3 as a therapeutic target for cancer and the tumor microenvironment. Arch Pharm Res 39: 1085-1099, 2016. 